\title{
Gamificando o ensino de programação de computadores: um Mapeamento Sistemático
}

\author{
Gustavo Steinmetz, Gustavo Lazarotto Schroeder, \\ Rosemary Francisco, Jorge Luis Victória Barbosa
}

\author{
Universidade do Vale do Rio dos Sinos (UNISINOS) \\ Av. Unisinos, 950 - Cristo Rei, São Leopoldo - RS, 93022-000 - Brazil \\ stzgustavo@gmail.com, gschroederdedu.unisinos.br, \\ \{rosemaryf, jbarbosa\}@unisinos.br
}

\begin{abstract}
The objective of this research is to identify studies that apply gamification to computer programming teaching. The study presents a systematic mapping that used six databases known in the academic field in computer science and software engineering, including publications between 2012 and 2021. After the refinements procedures, 17 articles were selected for analysis. The results show positive effects when using gamification in programming teaching, mainly related to motivation and engagement. Also, the studies observed better performance of the group of students who had contact with gamification due to the greater willingness of these students to seek knowledge.
\end{abstract}

Resumo. O objetivo desta pesquisa é identificar estudos que aplicam a gamificação ao ensino de programação de computadores. O estudo apresenta um mapeamento sistemático que utilizou seis bases de dados conhecidas no âmbito acadêmico em informática e engenharia de software, incluindo publicações entre 2012 e 2021. Após os procedimentos de refinamento, 17 estudos foram selecionados para análise. Os resultados indicam efeitos positivos do uso da gamificação no ensino de programação, principalmente relacionados à motivação e engajamento. Além disso, os estudos observaram melhor desempenho do grupo de alunos que tiveram contato com a gamificação devido à maior disposição desses alunos em buscar o conhecimento.

\section{Introdução}

A programação de computadores é uma das competências técnicas mais demandadas atualmente e que permite aumentar as chances de sucesso dos alunos no novo mundo digital [Akcaoglu et al. 2021]. Entretanto, aprender a programar é uma tarefa complexa, o que torna o processo de ensino e aprendizagem de programação um desafio para alunos e professores envolvidos no processo. Por conseguinte, é fundamental motivar e estimular o aluno por meio de mecanismos que suportem adequadamente esse processo através de abordagens que vão além dos métodos tradicionais que se materializam nas aulas [Schez-Sobrino et al. 2020], sejam elas presenciais ou virtuais.

Uma possível abordagem que pode ser utilizada para aumentar a motivação dos alunos ao aprender programação de computadores é a gamificação. A gamificação pode ser definida como o uso de elementos do design de jogos em contextos não relacionados 
a jogos [Deterding et al. 2011] a fim de impactar positivamente a motivação pessoal no contexto gamificado [DuVernet and Popp 2014]. A gamificação tem ganhado cada vez mais destaque no domínio educacional, principalmente devido ao seu uso para o aumento de motivação, interesse e desempenho do aluno na aprendizagem [Bachtiar et al. 2018]. Para enfatizar o crescimento e a relevância da gamificação no mundo acadêmico uma pesquisa foi realizada utilizando o motor de busca de literatura científica Google Scholar 1 o qual revela que nos documentos relativos ao ano de 2010 o termo "gamification" aparece apenas 145 vezes, enquanto no ano de 2020 o termo aparece 22.800 vezes.

Também se identificou um avanço de pesquisas relacionadas ao presente estudo, mapeamentos sistemáticos que observaram o uso da gamificação na educação de maneira geral [Borges et al. 2013, De Avila dos Santos and De Freitas 2017], em softwares educativos [Peixoto et al. 2015] e nos processos da engenharia de software [Belinazo and Fontoura 2017]. Outras três pesquisas realizaram mapeamentos no domínio do ensino da programação, porém com um foco diferente deste estudo. Silva, Fernandes e Santos (2018) mapearam o emprego de jogos digitais no ensino da programação de computadores, no cenário nacional. Inclusive como trabalho futuro, os autores recomendaram a realização de uma investigação mais abrangente olhando o cenário internacional, sendo esta recomendação o principal objetivo da presente pesquisa.

Shahid et al. (2019) e Pinheiro e De Sousa (2020), por sua vez, realizaram um mapeamento tendo como foco a identificação dos principais conceitos de programação e elementos de jogos adotados no suporte ao ensino destes conceitos. A contribuição da presente pesquisa se soma aos achados destes trabalhos, visto que, além da inclusão de outras bases de dados científicas e um período maior de observação, também considerouse outras questões como quais as teorias de aprendizagem estão sendo mais utilizadas em conjunto com a gamificação no ensino da programação de computadores.

Considerando esta notória relevância da gamificação no ambiente educacional, o presente trabalho teve como objetivo identificar estudos que envolvam a aplicação de gamificação no ensino de programação de computadores no cenário internacional. Para tanto, realizou-se um mapeamento sistemático da literatura. Observou-se na literatura que a gamificação tem contribuído para o ensino de programação de computadores, no entanto mais pesquisas são necessárias para melhor entender como a gamificação promove o aprendizado [Rodrigues et al. 2021]. Este entendimento possibilitará conhecer as tecnologias, técnicas e resultados relacionados ao tema, de modo a construir uma referência para pesquisas futuras.

Este artigo está organizado em quatro seções. A seção 2 descreve a metodologia utilizada no mapeamento sistemático. Na seção 3 são apresentados e discutidos os resultados. Finalmente, a seção 4 aborda as considerações finais.

\section{Metodologia do Mapeamento Sistemático}

O mapeamento sistemático utiliza a metodologia cuja execução envolve os seguintes passos [Petersen et al. 2008, Petersen et al. 2015]: 1) definir as questões de pesquisa; 2) definir o processo de pesquisa; 3) definir os critérios para a seleção dos artigos e 4) executar as análises e classificar os artigos selecionados.

\footnotetext{
${ }^{1}$ https://scholar.google.com/
} 


\subsection{Questões de Pesquisa}

Para guiar o processo de pesquisa foram definidas as Questões Gerais (QG), Questões Focais $(\mathrm{QF})$ e Questões Estatísticas $(\mathrm{QE})$ conforme apresenta a Tabela 1.

Tabela 1. Questões de pesquisa

ID Questão

QG1 Quais atividades educacionais no ensino de programação de computadores foram gamificadas?

QG2 Quais teorias e abordagens de aprendizagem foram aliadas à gamificação?

QG3 Quais são os principais impactos do uso de gamificação no ensino de programação de computadores?

QF1 Quais foram as técnicas utilizadas para incorporar gamificação no ensino de programação de computadores?

QF2 Quais limitações foram encontradas ao fazer o estudo de gamificação no ensino de programação de computadores?

QF3 Quais resultados foram identificados ao usar gamificação no ensino de programação de computadores?

QE1 Qual a distribuição dos artigos selecionados por base de dados a cada ano?

\subsection{Processo de Pesquisa}

O processo de pesquisa consistiu em: 1) especificar a string de busca; 2) escolher as bases de dados para realizar a busca; 3) executar a busca nas bases selecionadas e 4) obter os resultados. A string aplicada na busca foi a seguinte: ("education platform" OR "education system") AND ("programming" OR "software development" OR "software engineering") AND ("gamification" OR "gamify" OR "gamified" OR "gamifying”). Foram pesquisados os sinônimos para a obtenção de um resultado mais abrangente, possibilitando, desta forma, a localização de mais trabalhos relacionados ao tema.

As bases de dados selecionadas para a pesquisa foram: ACM Digital Library ${ }^{2}$, IEEE Xplore Digital Library ${ }^{3}$, Springer Link ${ }^{4}$, Wiley Online Library ${ }^{5}$, Science Direct ${ }^{6}$ e Scopus ${ }^{7}$. Tais bases de dados destacam-se como referências na área de computação.

\subsection{Filtros de Resultados}

Esta etapa define um processo de triagem para selecionar trabalhos relevantes, tendo Critérios de Inclusão (CI) e os Critérios de Exclusão (CE) definidos para orientar esta seleção. Os Critérios de Inclusão definem quais artigos devem ser incluídos no escopo,

\footnotetext{
${ }^{2}$ https://dl.acm.org/

${ }^{3}$ https://ieeexplore.ieee.org/

${ }^{4}$ https://link.springer.com/advanced-search

${ }^{5}$ https://onlinelibrary.wiley.com/

${ }^{6} \mathrm{https}: / /$ www.sciencedirect.com/

${ }^{7}$ https://www.scopus.com/
} 
enquanto os Critérios de Exclusão definem o que torna um estudo inelegível. As etapas a seguir foram executadas com base nos seguintes critérios de inclusão: 1) conteúdo completo à disposição; 2) trabalhos publicados entre 2012 e Março de 2021; 3) trabalhos na área de engenharia de software e educação; 4) trabalhos com o foco no uso da gamificação para o ensino de programação de computadores. Em contraponto, foram considerados os seguintes critérios de exclusão: 1) idioma diferente do Inglês, visto que o objetivo do artigo é observar o cenário internacional; 2) artigos duplicados e 3) livros, reviews e mapeamentos sistemáticos.

\section{Análise e discussão dos resultados}

A Figura 1 representa o processo de busca e filtragem realizado. Inicialmente a busca foi aplicada nos campos título, abstract e keywords, encontrando 4.450 artigos com base nas ferramentas de pesquisa das seis diferentes bases de dados. Tal consulta foi feita ao final de abril de 2021, usando a string de busca sem a aplicação de filtros.

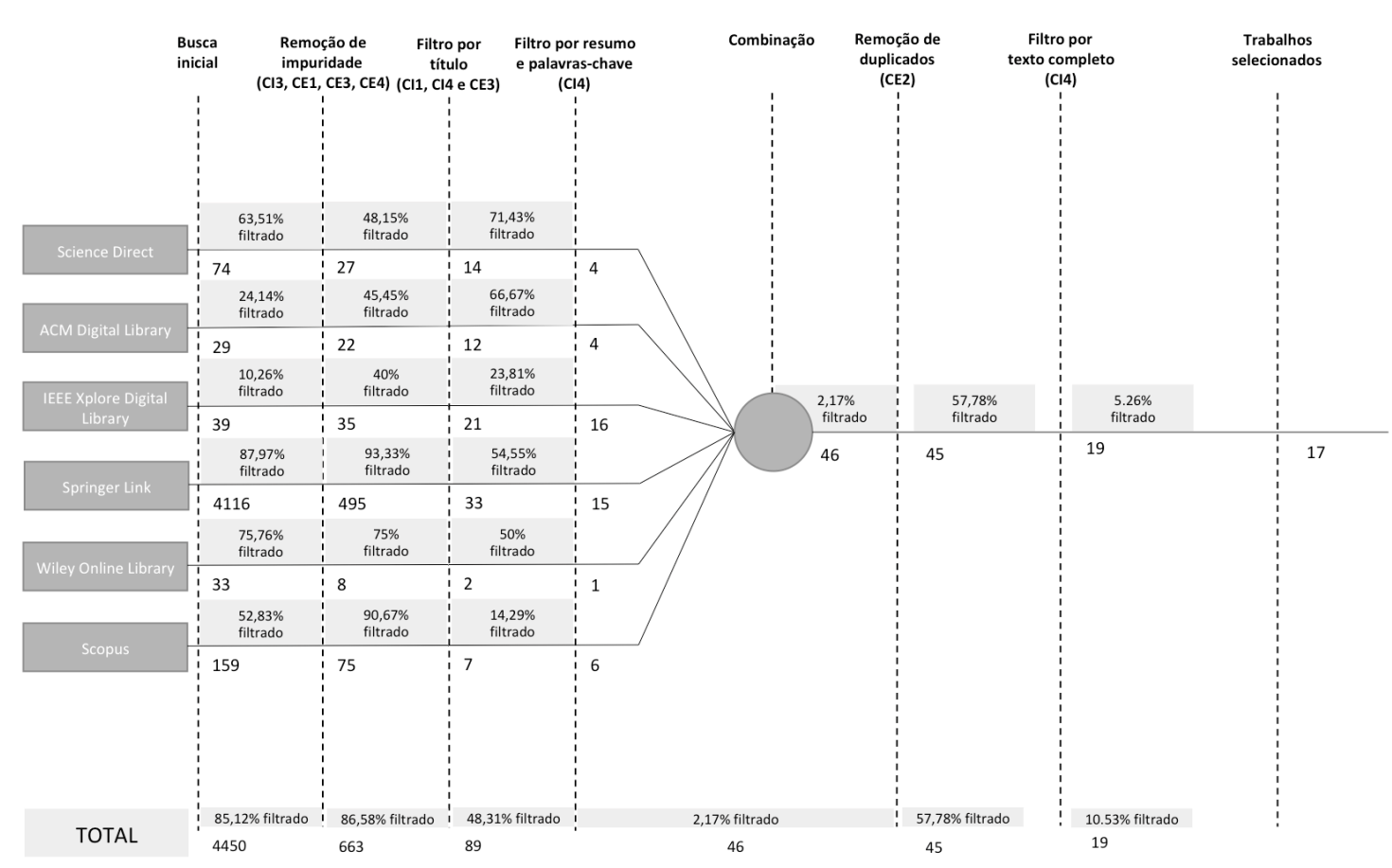

Figura 1. Resultado do filtro do mapeamento sistemático

Em seguida, na segunda etapa foi aplicado o filtro de inclusão CI3 e os filtros de exclusão CE1, CE3 e CE4, com o intuito de remover os trabalhos que não se enquadravam com o foco desta pesquisa. Com essa filtragem se obteve uma redução de $85,12 \%$, totalizando 663 artigos.

Após, foi iniciada a etapa responsável pela filtragem por título, na qual foram utilizados os filtros CI1, CI4 e CE3. Nesta etapa foram filtrados 86,58\% dos artigos, restando apenas 89. Na etapa seguinte foi aplicado o filtro por abstract e keywords, usando o filtro de inclusão CI4. 
Tabela 2. Lista de artigos selecionados

ID AUTORES/TÍTULO/PUBLICAÇÃO/ANO

1 [Seralidou, Eleni and Douligeris, Christos] Learning programming by creating games through the use of structured activities in secondary education in Greece (Education and Information Technologies) 2021

2 [Bernik, A. et al.] Introducing gamification into e-learning university courses (2017 40th International Convention on Information and Communication Technology, Electronics and Microelectronics, MIPRO 2017 - Proceedings) 2017

3 [Maarek, Manuel et al.] How Could Serious Games Support Secure Programming? Designing a Study Replication and Intervention (Proceedings - 4th IEEE European Symposium on Security and Privacy Workshops, EUROS and PW 2019) 2019

4 [Qu, Wei Qing et al.] Research on teaching gamification of software engineering (Proceedings of the 9th International Conference on Computer Science and Education, ICCCSE 2014) 2014

5 [Bachtiar, Fitra A et al.] CoMa : Development of Gamification-based (2018 10th International Conference on Information Technology and Electrical Engineering (ICITEE)) 2018

6 [Mi, Qing et al.] A Gamification Technique for Motivating Students to Learn Code Readability in Software Engineering (Proceedings - 2018 International Symposium on Educational Technology, ISET 2018) 2018

7 [Fuchs, Marks and Wolff, Christian] Improving programming education through gameful, formative feedback (IEEE Global Engineering Education Conference, EDUCON) 2016

8 [Berkling, Kay and Neubehler, Katja] Presenting an open-source platform for supporting gamified class teaching with peer reviews (IEEE Global Engineering Education Conference, EDUCON) 2019

9 [Issa, Lana and Jusoh, Shaidah] Usability evaluation on gamified e-learning platforms (ACM International Conference Proceeding Series) 2019

10 [Morales-Trujillo, Miguel Ehécatl and Garciá-Mireles, Gabriel Alberto] Gamification and SQL: An Empirical Study on Student Performance in a Database Course (ACM Transactions on Computing Education) 2021

11 [Schez-Sobrino, Santiago et al.] RoboTIC: A serious game based on augmented reality for learning programming (Multimedia Tools and Applications) 2020

12 [Lehtonen, Timo et al.] On the role of gamification and localization in an open online learning environment: javala experiences (ACM International Conference Proceeding Series) 2015

13 [Edwards, Stephen H. and Li, Zhiyi] A Proposal to Use Gamification Systematically to Nudge Students Toward Productive Behaviors (ACM International Conference Proceeding Series) 2020

14 [Clegg, Benjamin S. et al.] Teaching software testing concepts using a mutation testing game (Proceedings - 2017 IEEE/ACM 39th International Conference on Software Engineering: Software Engineering and Education Track, ICSE-SEET 2017) 2017

15 [Smiderle, Rodrigo et al.] The impact of gamification on students' learning, engagement and behavior based on their personality traits (Smart Learning Environments) 2020

16 [Yadav, Alok Kumar and Oyelere, Solomon Sunday] Contextualized mobile game-based learning application for computing education (Education and Information Technologies) 2020

17 [Rojas-López, Arturo et al.] Engagement in the course of programming in higher education through the use of gamification (Universal Access in the Information Society) 2019 
Ao final dessa etapa, foram filtrados 48,31\% dos artigos, restando um total de 46 trabalhos. Ao concluir as últimas etapas de filtragem, referente aos filtros de remoção de duplicados (CE2), filtro por texto completo (CI4) e seleção de trabalhos, chegando-se ao número de 17 artigos, sendo eles os presentes na Tabela 2. As seções a seguir apresentam os resultados e discussão com base em cada questão de pesquisa.

\section{1. (QG1) Quais atividades educacionais no ensino de programação foram gamificadas?}

Através dos estudos mapeados encontrou-se a gamificação sendo utilizada no ensino básico de programação ( 4 artigos, IDs $=1,2,5,11$ ), ensino da linguagem de programação JAVA ( 1 , ID = 5), ensino de linguagens de banco de dados como SQL (1, ID = 10) e ensino de legibilidade de código $(1, \mathrm{ID}=6)$. Entre os estudos que aplicaram gamificação no ensino básico de programação, destacam-se os trabalhos de Seralidou e Douligeris (2021) e de Schez-Sobrino et al. (2020), IDs 1 e 11 respectivamente.

Seralidou e Douligeris (2021) fizeram o uso de gamificação com o intuito de ensinar princípios básicos de programação, como condicionais, loops e variáveis para alunos do ensino médio e promover o pensamento crítico durante as tomadas de decisão, necessárias para as tarefas de programação. Já Schez-Sobrino et al. (2020) foram além da gamificação e construíram um jogo sério baseado em realidade aumentada. Por meio desta abordagem, os alunos vão passando por níveis e aprendendo programação de maneira incremental.

\section{2. (QG2) Quais teorias e abordagens de aprendizagem foram aliadas à gamificação?}

Foram observadas as seguintes teorias e abordagens de aprendizagem: teoria do flow (2, IDs $=2,17)$, teoria da autodeterminação $(1, \mathrm{ID}=6)$, teoria do esforço cognitivo e taxonomia dos objetivos educacionais $(1$, ID $=7)$, aprendizagem cooperativa $(1, I D=8)$, arquitetura da escolha e self-regulated learning ( 1 , ID = 13) e conectivismo (1, ID = 16).

Tanto a teoria do flow quanto a teoria da autodeterminação são bastante utilizadas em aplicações de gamificação. Os estudos referentes a teoria do flow foram derivados de pesquisas sobre o processo criativo, com o psicólogo húngaro Mihaly Csikszentmihalyi na década de 60. O estado de flow tem sido amplamente utilizado para descrever uma experiência ótima caracterizada como um estado de estar totalmente focado e envolvido em uma atividade [Csikszentmihalyi 1990].

Já a teoria da autodeterminação (Self-determination theory - SDT), proposta por Deci e Ryan (2000), identifica fatores que possam afetar a motivação, tanto positivamente como negativamente. A SDT define que o ser humano possui três necessidades psicológicas inatas que quando satisfeitas garantem o seu crescimento saudável e sua vitalidade [Deci and Ryan 2000].

\section{3. (QG3) Quais são os principais impactos do uso de gamificação no ensino de programação?}

Em uma análise empírica no ensino de "Programação II"em cursos universitários com $e$ Learning, percebeu-se que os alunos do grupo experimental os quais tiveram gamificação implementada em seu aprendizado, mostraram um interesse médio maior nas atividades 
disponíveis no curso online em comparação ao grupo controle (grupo que não teve uso de gamificação), como Fórum (5,6 vezes maior), Resultados de aprendizagem (9,7 vezes maior) e Lista de termos (43,3 vezes maior) $(1, \mathrm{ID}=2)$.

Apesar dos 17 estudos analisados indicarem efeitos positivos da aplicação de gamificação no ensino e aprendizagem, apenas o estudo mencionado acima traz detalhes acerca de seus impactos. Portanto, observa-se espaço para pesquisas mais profundas e longitudinais a fim de averiguar os impactos da gamificação no ensino e aprendizagem de programação de computadores [Rodrigues et al. 2021].

\section{4. (QF1) Quais foram as técnicas utilizadas para incorporar gamificação no ensino de programação?}

Foram encontradas diversas técnicas de gamificação no ensino de programação dentre elas: pontos de atividade ou experiência (11, IDs = 5, 6, 7, 8, 10, 12, 13, 14, 15, 16, 17), leaderboard ( 3 , IDs $=5,6,17$ ), ensino de programação através da criação de jogos (1, ID =1), plugin de gamificação para Moodle (1, ID = 2), Serious Games (3, IDs = 3, 11, 16), competição entre alunos através de confrontos em certas atividades (4, IDs =4, 5, 10, 14), badges (5, IDs $=5,6,12,15,17)$, nível de experiência $(5$, IDs $=5,7,12,13,14)$, barra ou status de progresso ( 3 , IDs $=7,8,13)$, feedback imediato ( 6 , IDs $=7,8,9,13,16,17$ ), jogo de interpretação de papéis, também conhecido como RPG $(1, I D=13)$, energia de submissão, ou seja, limitação de ações por dia (1, ID = 13).

Entretanto, a fim de promover os benefícios motivacionais não basta que a aplicação use apenas recompensas extrínsecas como pontos ou badges, é importante que os problemas sejam bem ordenados durante a instrução. A gamificação possui mecânicas e dinâmicas, as mecânicas especificam regras, objetivos, configurações e interações do jogo [Robson et al. 2015]. Essas mecânicas tendem a fornecer estrutura às atividades, ao invés de estimular os usuários a explorar e experimentar de forma independente. Já as dinâmicas definem o tipo de comportamento que surgem nos jogadores quando os mesmos participam da experiência gamificada.

As mecânicas de jogos podem ser divididas em comportamento, feedback e promoção. De acordo com a categorização de Bartle (1996), a personalidade pode ser dividida em subtipos vencedor, locutor, colecionador e pesquisador. A Tabela 3 mostra os 16 mecanismos de gamificação que foram citados com mais frequência nos trabalhos analisados.

Tabela 3. Mecanismos de gamificação mais apresentados na literatura

\begin{tabular}{|c|c|c|c|}
\hline Conquistas & Bônus & $\begin{array}{c}\text { Contagem } \\
\text { Regressiva }\end{array}$ & Badges \\
\hline Desafios & Onboarding & Efeito "Combo" & Níveis \\
\hline Impulsividade & Easter Eggs & $\begin{array}{c}\text { Significado } \\
\text { épico }\end{array}$ & Perda da aversão \\
\hline Status Social & $\begin{array}{c}\text { Recompensas } \\
\text { Programadas }\end{array}$ & Recompensas Aleatórias & Pontos \\
\hline
\end{tabular}




\section{5. (QF2) Quais limitações foram encontradas ao fazer o estudo de gamificação no ensino de programação?}

Em geral, cada pessoa tem seu próprio método de reconhecimento e processamento de informações e essa diferença no aprendizado também se aplica aos alunos. Tendo em conta esta forma de aprendizagem personalizada e adaptativa, uma abordagem construtivista passo a passo pode não ser adequada para a aprendizagem em todos os casos (1, ID $=1)$.

Vale ressaltar que a maioria dos estudos relataram uma limitação similar referente a representatividade da amostra dos grupos de pesquisa, por exemplo, "todos os participantes que ingressaram no estudo são da mesma universidade, a maioria jovens. Portanto, não é razoável generalizar os resultados para toda a população estudantil. Estatisticamente, isso pode ser resolvido com a replicação desse experimento em amostras distintas de alunos de graduação" $(1, \mathrm{ID}=15)$.

\section{6. (QF3) Quais resultados foram identificados ao usar gamificação no ensino de programação?}

Os artigos evidenciam que o uso da gamificação aumenta o engajamento da maioria dos alunos, consequentemente aumentando o desempenho do grupo de alunos. Percebeuse também que ao comparar dois grupos, um experimental com gamificação no ensino e outro grupo controle com educação convencional, os alunos do grupo experimental usaram materiais didáticos e bônus 3,69 vezes mais do que o grupo controle ( 1 , ID =2).

Foi identificado em um estudo com um grupo gamificado e outro grupo controle sem gamificar, que alunos introvertidos que usaram a versão gamificada foram mais engajados do que os alunos extrovertidos para a mesma versão. Foi encontrada uma forte correlação negativa entre o traço de extroversão e o número de visualizações de classificação, indicando que a gamificação em geral e, especialmente o elemento de classificação, é mais benéfica para os introvertidos $(1$, ID = 15). Devido aos resultados positivos atrelados ao uso de gamificação no ensino de programação nos trabalhos selecionados, pode-se assumir estatisticamente que a gamificação está positivamente relacionada ao ensino da programação.

\section{7. (QE1) Qual a distribuição dos artigos selecionados por base de dados a cada ano?}

A Figura 2 apresenta uma representação visual com os artigos classificados por base de dados e uma linha do tempo de 2012 até Março de 2021 com a distribuição dos artigos por ano. Nota-se que há uma crescente no número de artigos relacionados ao tema. A queda no final no gráfico se deve a este estudo ter conduzido a busca de trabalhos no mês de março, entretanto a quantidade de artigos é similar ao ano anterior. Isso pode indicar uma tendência de crescimento na quantidade de artigos até o fim de 2021, seguindo o padrão de aumento de artigos visto nos anos anteriores.

Além disso percebe-se que a base de dados com maior quantidade de artigos tratados nesse mapeamento foi a IEEE, com 7 artigos, seguido da Springer Link e a Scopus, ambas com 4 artigos selecionados, por fim, a ACM com 2 artigos. As bases Wiley e Science Direct não tiveram artigos selecionados. 


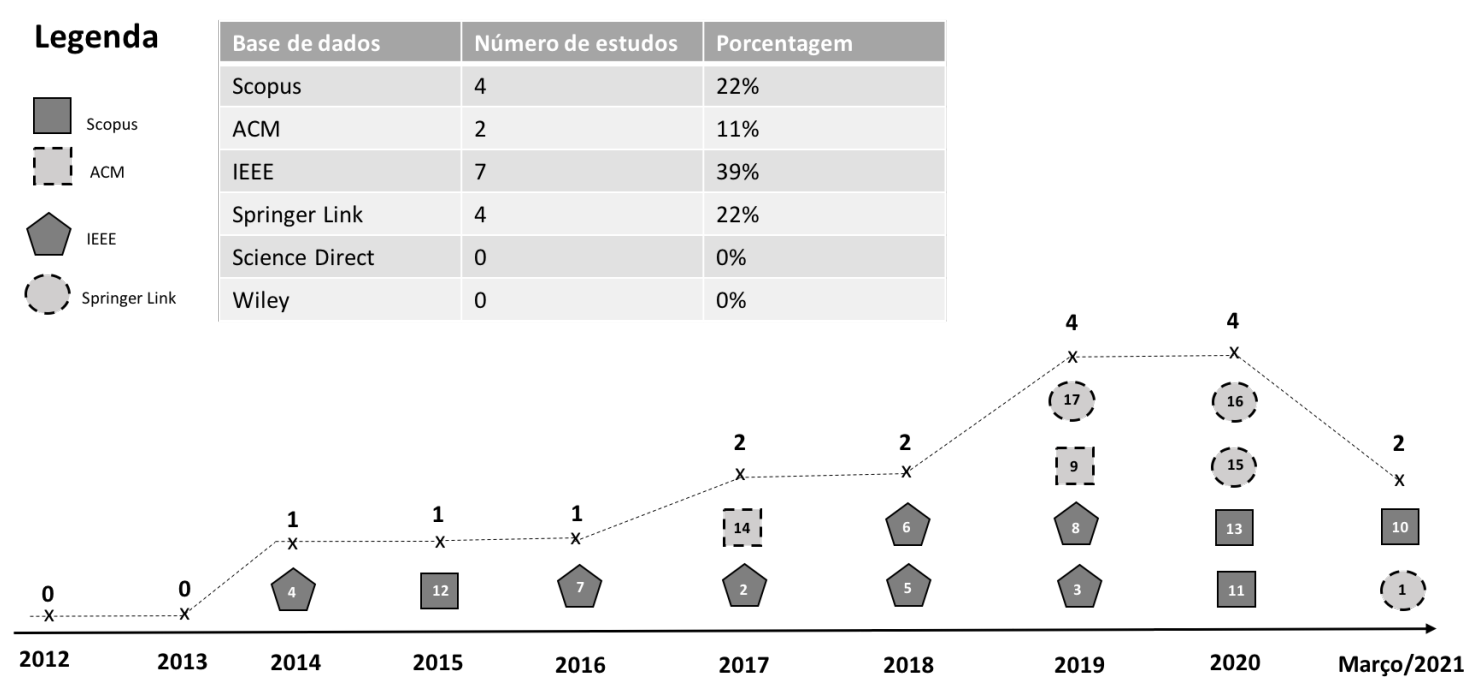

Figura 2. Distribuição dos artigos por ano.

\section{Considerações Finais}

Este artigo apresenta o resultado de um mapeamento sistemático no qual foram analisados 17 artigos envolvendo o uso da gamificação no ensino da programação de computadores. O estudo identificou um aumento crescente em estudos relacionados ao uso de gamificação para o ensino da programação em diversos aspectos, desde o ensino básico de programação até tópicos avançados como legibilidade de código. Além disso, o estudo reforça e evolui as contribuições de outras pesquisas já realizadas nesta temática [Silva et al. 2018, Shahid et al. 2019, Pinheiro and Sousa 2020].

Embora os artigos reforcem que a gamificação está positivamente relacionada com o ensino da programação uma limitação exposta foi a dificuldade de generalizar os resultados, visto que, cada pessoa tem seu próprio método de reconhecimento e processamento de informações, em contramão a esta limitação apenas um artigo relatou a utilização de personalização da gamificação por tipo de jogadores [Bartle 1996]. Pontos de atenção foram encontrados, uma vez que as técnicas mais utilizadas para a gamificação do ensino de programação foram Points, Badges e Leaderboards (PBL) (76,48\%, 13/17). Apesar de PBL ser uma abordagem comum para a gamificação, deve-se ter cuidado ao utilizar pois seu uso pode causar efeitos negativos sobre a motivação intrínseca dos alunos [Deci et al. 1999, Landers et al. 2017, Aldemir et al. 2018].

Finalmente, observou-se que embora poucos artigos tenham discutido impactos e resultados do uso de gamificação no ensino da programação de computadores, todos os trabalhos que mencionaram este tópico reportaram haver relação positiva entre a gamificação e o interesse, engajamento e desempenho dos alunos. Constatou-se também que alunos com maior traço de introversão tiveram maior benefício em relação a estudantes extrovertidos. Sendo assim, o resultado deste trabalho incentiva pesquisadores a explorarem frameworks como a escala Hexad [Tondello et al. 2016], a fim de estabelecer uma relação entre as técnicas utilizadas para a gamificação e o público alvo da aplicação [Werbach and Hunter 2012, Tondello et al. 2016].

Como trabalhos futuros sugere-se uma abordagem gamificada de ensino de 
programação de computadores, que permita a personalização do processo educativo com base no perfil de cada aluno. As pesquisas futuras podem aplicar análise de perfis de usuários [Tondello et al. 2016] e mecânicas de gamificação adequadas a cada perfil de aluno [Marczewski 2015]. Além disso, trabalhos futuros podem explorar melhor o uso de frameworks de gamificação [Werbach and Hunter 2012, Chou 2019] para o desenvolvimento da atividade educacional gamificada.

\section{Referências}

Akcaoglu, M., Rosenberg, J. M., Hodges, C. B., and Hilpert, J. C. (2021). An exploration of factors impacting middle school students' attitudes toward computer programming. Computers in the Schools, 38(1):19-35.

Aldemir, T., Celik, B., and Kaplan, G. (2018). A qualitative investigation of student perceptions of game elements in a gamified course. Computers in Human Behavior, 78:235-254.

Bachtiar, F. A., Pradana, F., Priyambadha, B., and Bastari, D. I. (2018). Coma: Development of gamification-based e-learning. In 2018 10th International Conference on Information Technology and Electrical Engineering (ICITEE), pages 1-6.

Bartle, R. (1996). Hearts, clubs, diamonds, spades: Players who suit muds. Journal of MUD research, 1(1):19.

Belinazo, G. and Fontoura, L. (2017). Mapeamento sistemático de gamificação em engenharia de software. In Anais da I Escola Regional de Engenharia de Software, pages 193-202, Porto Alegre, RS, Brasil. SBC.

Borges, S. D. S., Reis, H. M., Durelli, V. H. S., Bittencourt, I. I., Jaques, P. A., and Isotani, S. (2013). Gamificação Aplicada à Educação: Um Mapeamento Sistemático. In Brazilian Symposium on Computers in Education (Simpósio Brasileiro de Informática na Educação-SBIE).

Chou, Y.-k. (2019). Actionable gamification: Beyond points, badges, and leaderboards. Packt Publishing Ltd.

Csikszentmihalyi, M. (1990). Flow: The psychology of optimal experience. Harper \& Row New York.

De Avila dos Santos, J. and De Freitas, A. L. C. (2017). Gamificação Aplicada a Educação: Um Mapeamento Sistemático da Literatura. RENOTE, 15(1).

Deci, E. L., Koestner, R., and Ryan, R. M. (1999). A meta-analytic review of experiments examining the effects of extrinsic rewards on intrinsic motivation. Psychological bulletin, 125(6):627.

Deci, E. L. and Ryan, R. M. (2000). The"what"and"why"of goal pursuits: Human needs and the self-determination of behavior. Psychological inquiry, 11(4):227-268.

Deterding, S., Khaled, R., Nacke, L. E., Dixon, D., et al. (2011). Gamification: Toward a definition. In CHI 2011 gamification workshop proceedings, volume 12, pages 12-15. Vancouver BC, Canada.

DuVernet, A. M. and Popp, E. (2014). Gamification of workplace practices. The Industrial-Organizational Psychologist, 52(1):39-44. 
Landers, R. N., Bauer, K. N., and Callan, R. C. (2017). Gamification of task performance with leaderboards: A goal setting experiment. Computers in Human Behavior, 71:508515 .

Marczewski, A. (2015). Even ninja monkeys like to play. London: Blurb Inc.

Peixoto, M., Silva, C., Vilela, J., and Gonçalves, E. (2015). Um Mapeamento Sistemático de Gamificação em Software Educativo no Contexto da Comunidade Brasileira de Informática na Educação. In Workshop de Informática na Escola, page 584.

Petersen, K., Feldt, R., Mujtaba, S., and Mattsson, M. (2008). Systematic Mapping Studies in Software Engineering. 12th International Conference on Evaluation and Assessment in Software Engineering, pages 68-77.

Petersen, K., Vakkalanka, S., and Kuzniarz, L. (2015). Guidelines for conducting systematic mapping studies in software engineering: An update. Information and Software Technology, 64:1-18.

Pinheiro, A. F. and Sousa, R. R. (2020). Uma revisão sistemática do uso de elementos dos jogos para motivar os estudantes para o aprendizado de programação. Brazilian Journal of Development, 6(6):36612-36636.

Robson, K., Plangger, K., Kietzmann, J. H., McCarthy, I., and Pitt, L. (2015). Is it all a game? understanding the principles of gamification. Business horizons, 58(4):411420.

Rodrigues, L., Toda, A. M., Oliveira, W., Palomino, P. T., Avila-Santos, A. P., and Isotani, S. (2021). Gamification Works, but How and to Whom? An Experimental Study in the Context of Programming Lessons, page 184-190. Association for Computing Machinery, New York, NY, USA.

Schez-Sobrino, S., Vallejo, D., Glez-Morcillo, C., Redondo, M., and Castro-Schez, J. J. (2020). RoboTIC: A serious game based on augmented reality for learning programming. Multimedia Tools and Applications, 79(45-46).

Shahid, M., Wajid, A., Haq, K. U., Saleem, I., and Shujja, A. H. (2019). A Review of Gamification for Learning Programming Fundamental. In 2019 International Conference on Innovative Computing (ICIC), pages 1-8. IEEE.

Silva, R. R., Fernandes, J., and Santos, R. (2018). Panorama da Utilização de Jogos Digitais no Ensino de Programação no Nível Superior na Última Década: Uma Revisão Sistemática da Literatura. In Brazilian Symposium on Computers in Education (Simpósio Brasileiro de Informática na Educação-SBIE), page 535.

Tondello, G. F., Wehbe, R. R., Diamond, L., Busch, M., Marczewski, A., and Nacke, L. E. (2016). The gamification user types hexad scale. In Proceedings of the 2016 annual symposium on computer-human interaction in play, pages 229-243.

Werbach, K. and Hunter, D. (2012). For the win: How game thinking can revolutionize your business. Wharton digital press. 